\title{
Anti-acceleration Disturbance Method for Pendulum Tiltmeter
}

\author{
Hui Zhao ${ }^{1, a}$, FuxueZhang ${ }^{2, b}$, Qingwen Yan ${ }^{1, c}$, Xing Gao ${ }^{2, d}$ \\ ${ }^{1}$ School of electronic Engineering, Beijing University of Posts and Telecommunications,Beijing \\ 100876,China \\ ${ }^{2}$ Sensing Technique Research Center, Beijing Information Science and Technology University, \\ Beijing 100101, China \\ aasdfzh@163.com, bzhangfuxue@263.net, 'quingwenxp@yahoo.com.cn, dpigpigv@126.com
}

Keywords: pendulum tiltmeter; anti-acceleration disturbance; angular acceleration.

Abstract. With the practical application of silicon micro-machined gyro driven by rotation aircraft, it is found that the rotation aircraft's rudder surface vibration significantly influences the performance of the gyro. In this paper, as the background of the silicon micro-machined gyro, the vibration mechanism is analyzed according to the mathematics model and dynamics. Under the influence of vibration, both of the silicon pendulum's stress form and output signal are obtained. The paper proposes a reasonable installation method to suppress the vibration. The experimental result shows that the method is effective to reduce the gyro's output signal noise. And the result is good.

\section{Introduction}

Pendulum type tiltmeter has high accuracy in static test, but it is susceptible to the disturbance of linear acceleration and angular acceleration [1]. Experience of test shows that linear acceleration and angular acceleration disturbs pendulum tiltmeter in different way: the disturbance of linear acceleration is devastative, and the output of pendulum tiltmeter will be seriously distorted. When carrier vector angle changes without linear acceleration exist (called dynamic process), the test accuracy of the tilt sensor is decline. Because the disturbance of linear acceleration is much bigger comparative, the past research is concentrated on linear acceleration.

Based on the structure and principle of the pendulum tiltmeter, the reason that linear acceleration and angular acceleration disturbs pendulum type tiltmeter in different way is analyzed, and present corresponding scheme to restrain the disturbance of acceleration. The scheme can identify linear acceleration sensitively, and control the disturbance in a short time. In addition, the scheme can restrain angular acceleration disturbance effectively.

\section{Acceleration Disturbance on Pendulum Tiltmeter}

According to the type of medium, pendulum tiltmeter can be divided into solid pendulum tiltmeter, liquid pendulum tiltmeter and gas pendulum tiltmeter. All of the pendulums tiltmeter use character of pendulum in the gravitational field as well as Newton's second law [2-5]. For tiltmeter, gravity is a acceleration with constant magnitude and direction, so any other acceleration forced on the pendulum will disturb detection of pendulum tiltmeter. And because three types pendulum tiltmeter have same detection basis, the character of acceleration disturbance will be similar. Then acceleration disturbance on solid pendulum tiltmeter will be researched, as an example.

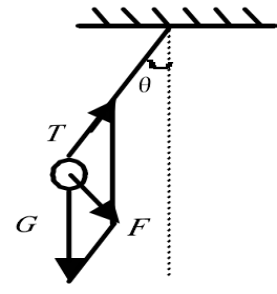

(a) Solid pendulum

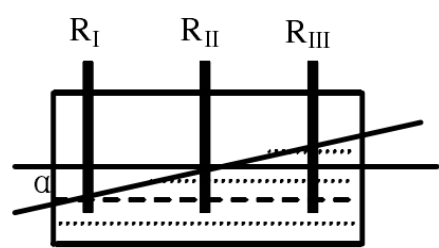

(b) Liquid pendulum
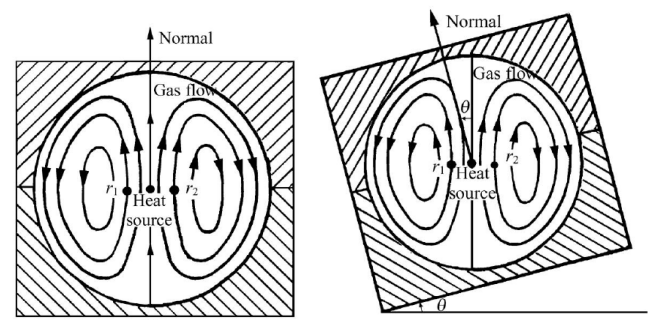

(c) Gas pendulum 
Figure 1. Principle of pendulum tiltmeter

As shown in Fig.1(a), force balance servo system is widely used in solid pendulum tiltmeter which composed by pendulum, cycloid, bracket, pendulum subject to gravity $\mathrm{G}$, tension $\mathrm{T}$ and their combined force $\mathrm{F}$ is:

$F=G \sin \theta=m g \sin \theta$

$\theta$ is the angle between the cycloid and the vertical direction. In small-angle range, relationship between $\mathrm{F}$ and $\theta$ is linear. Other solid pendulum tiltmeter including strain gauge type, potentiometer type, inductive type, vibrating-wire type and force-balance tilt sensor are also based on the principle.

The (1) will no longer valid with any acceleration exists. But the influences of linear acceleration and angular acceleration are different. Linear acceleration disturbance is destructive. The reason is the direction of linear acceleration could be in any direction, so force balance shown in Fig.1(a) will be destructed seriously and (1) cannot be valid. Then pendulum tiltmeter signal will be seriously distorted.

In dynamic process, angular acceleration direction will be same as or adverse to the direction of $F$. The direct range of pendulum tiltmeter is small. Then usually the magnitude of angular acceleration which proceeds from angular change in a small-angle range is small. For the two reasons above, angular acceleration disturb pendulum tiltmeter is minor relatively.

As showed in Fig.1(b) and Fig.1(c), liquid pendulum tiltmeter and gas pendulum tiltmeter also use gravity in angular detection. So they have similar characters of acceleration disturbance to solid pendulum tiltmeter. For the devastative effects of linear acceleration, pendulum tiltmeter signal should not be used when any linear acceleration exist. And angular acceleration reduces detection accuracy of the pendulum tiltmeter.

\section{Anti-acceleration Disturbance Method}

Structure of Prototype.The test prototype consists of pendulum tiltmeter, angular rate gyro, microcontroller, $\mathrm{AD}$ chip and other peripheral components. The detailed information of main components are shown in Tab.1.

TABLE I. INFORMATION OF COMPONENTS

\begin{tabular}{|c|c|c|}
\hline \multirow{2}{*}{ components } & \multicolumn{2}{|c|}{ detailed information } \\
\cline { 2 - 3 } & Version & company \\
\hline Pendulum iltmeter & SCA103T-D05 & VTI \\
\hline Angular rate gyro & LCG50 & BEI \\
\hline MCU & MSC1214Y3 & TI \\
\hline AD chip & AD7656 & ADI \\
\hline
\end{tabular}

The sensitive direction of the tiltmeter and angular rate gyro should coincide with each other. Analog signals of tiltmeter and gyro are sampled by AD chip and processed by MCU. After some digital data processing, the MCU output the final angle signal in analog voltage.

For sensitive direction of the tiltmeter and gyro is coinciding with each other, in a short time, angular variation sensed by tiltmeter should equate to the integration of angle velocity sensed by gyro in dynamic process. This feature will be applied into linear acceleration disturbance suppression.

Before dynamic detection, some kinds of static test and adjustment should be done, including: the zero voltage of tiltmeter test and gyro zero voltage test, linearity compensation, DA output range adjustment, and so on. Static adjustment objectives are: non-linearity $<1 \% \mathrm{FS}$, resolution is $0.01^{\circ}$.

Detection and Suppress the Disturbance of Linear Acceleration.For destructive impact on tiltmeter, linear acceleration must be detected. Angular rate gyro is sensitive to angular velocity, and angular acceleration can also be sensed indirectly. According to classical physics, the change in vector angle should be equal to the integral of the vector angular velocity in corresponding period.

When the disturbance of the linear acceleration does not exist, in a short time, the angle change amount detected by tiltmeter and gyro should be the same. The angle change amount is:

$$
V_{\theta T}-V_{\theta 0}=K_{\theta} \int_{0}^{T} \frac{V_{\omega}-V_{\omega 0}}{K_{\omega}} d t
$$


where $K_{\theta}$ is scale factor of the tiltmeter, and $K_{\omega}$ is the scale factor of gyro. $V_{\theta 0}$ is zero voltage of the tiltmeter, and $V_{\omega 0}$ is zero voltage of the gyro. $V_{\theta T}$ and $V_{\omega}$ are the voltage of tiltmeter and gyro.

The (2) cannot be used in digital data processing. When $T$ equate to the sample cycle of AD chip, the (2) can be turned to be (3) as follow.

$$
Q_{\theta T}-Q_{\theta 0}=K_{1} \frac{\left(T_{n}+T_{n-1}-2 T_{0}\right)}{2} \Delta t
$$

Where $\Delta t$ is sample cycle of the AD chip; $T_{\mathrm{n}-1}$ and $T_{\mathrm{n}}$ is the sample data of gyro, $T_{0}$ is the sample data of gyro zero. $Q_{\mathrm{n}-1}$ and $Q_{\mathrm{n}}$ is the sample data of tiltmeter. $K_{1}$ is scale factor between tiltmeter data and gyro data.

When tiltmeter is disturbed by linear acceleration, the left side of (3) will change faster than the right side. The difference $C a$ is:

$$
C \mathrm{a}=Q_{\theta T}-Q_{\theta 0}-K_{1} \frac{\left(T_{n}+T_{n-1}-2 T_{0}\right)}{2} \Delta t
$$

A constant value $M$ should be use. When the absolute value of $\mathrm{Ca}$ is bigger than $\mathrm{M}$, linear acceleration is existing. And in that case, angle change amount should be detected by integral of angular rate other than tiltmeter signal.

Because Integral error will increase with time, this method of calculation can only be used in a short time.

Elimination the Disturbance of Angular Acceleration.High accuracy turntable is used to implement the dynamic process. Because the accuracy of turntable is much higher than tiltmeter, the data output by turntable is consided to be the real angle of prototype. Then deviation of tiltmeter could be test.

The test method is: install the prototype in the middle frame of three-axis turntable. After sample, smoothing, linearization and other process, angle signal and angular rate signal of prototype is output in analog voltage. The voltages are caught by a independent digital device which receives turntable synchronously.

The middle frame of three-axis turntable worked in angle vibration mode, the angle range is from $-20^{\circ}$ to $20^{\circ}$, and vibration cycle is 5 s. One cycle output voltage of tiltmeter and gyro is shown in Fig.2, and the synchronous angle of turntable is shown in Fig 3.

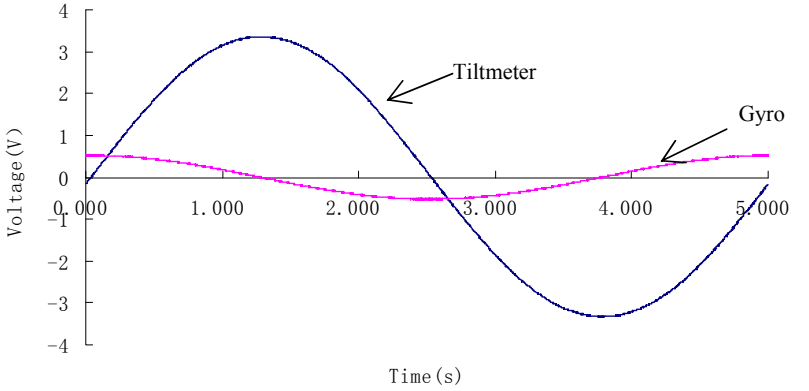

Figure 2. Analog output of tilt and gyro

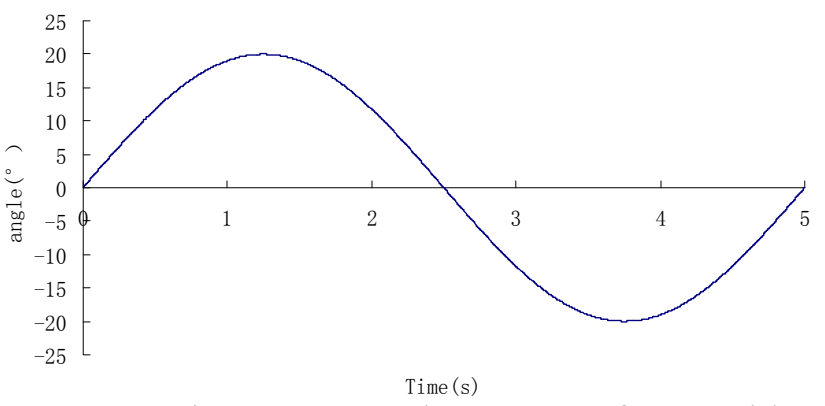

Figure 3. Angular output of Turntable

After converting angle value of turntable into voltage value, deviation value could be calculated by comparing the voltages of tiltmeter and turntable. The deviation curve is shown in Fig. 4.

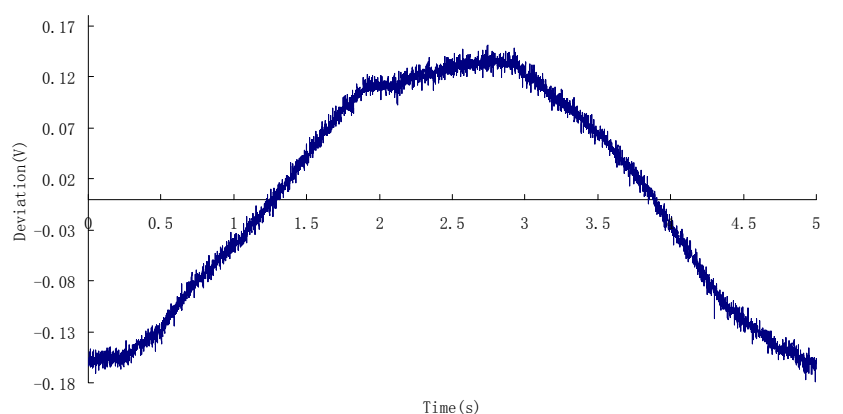

Figure 4. Deviation voltage 
The curve shown in Fig.4 has been test for several times, and obtains the same deviation curve. But the noise in value of deviation and gyro disturb the subsequent research seriously. Because the change rate of deviation value and the angular acceleration are small. Then divide the one cycle into two sections, and use three-order polynomial fitting to obtain smooth data curve。

Then change rate of deviation could be calculated. The relationship between angular rate and change rate of deviation is shown in Fig. 5.

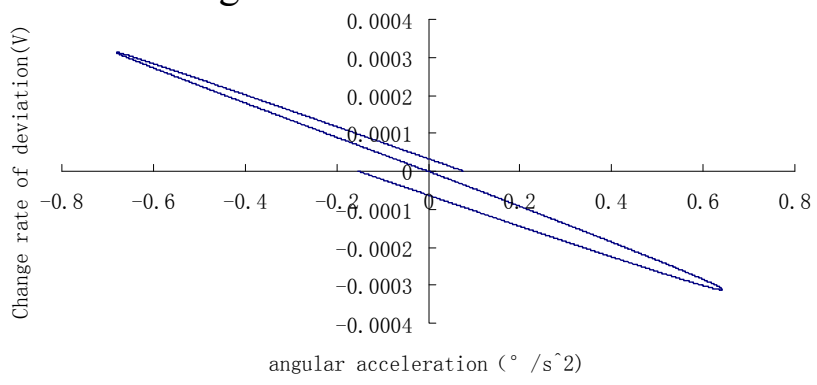

Figure 5. Curve of whole cycle

The curve in Fig.5 is not closed, that means that there are some problems in data acquisition or processing of deviation and gyro sampled data.

However, the relationship should be linear. To chose a half-cycle of data from the minimum of the angular acceleration. The curve of selected values is shown in fig.6.

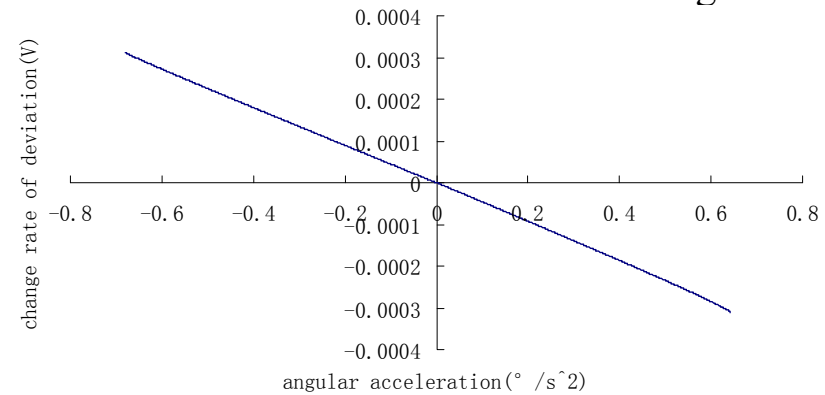

Figure 6. Curve of selected half cycle

As shown in Fig.6, a linear relationship between change rate of deviation and angular acceleration. According to this linear relationship, disturbance can be calculated by angular acceleration sensed by gyro indirectly. Then the disturbance of angular acceleration can be eliminated. signal minus the amount of error can be obtained by the real angle signal.

Main Part of Data Processing.The main part of data processing is shown in Fig.7.

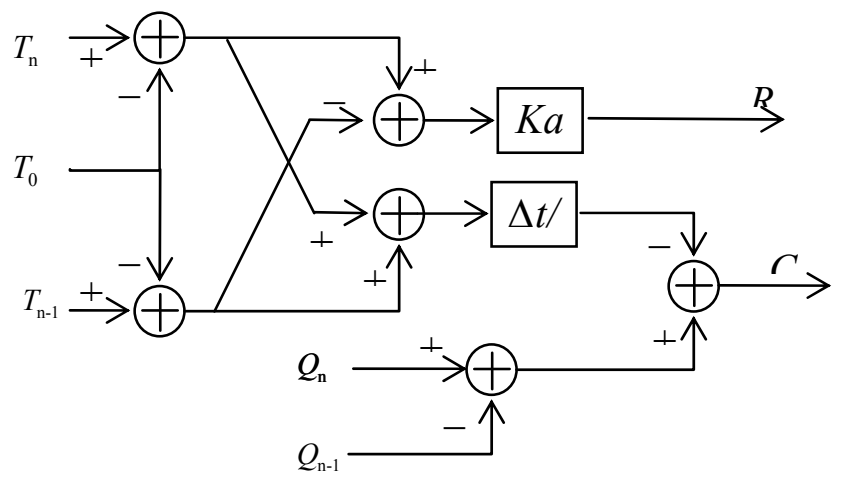

Figure 7. Main part of data process

Where $\mathrm{Ka}$ is the scale factor between change rate of tiltmeter deviation and angular acceleration; $R a$ is deviation caused by angular acceleration;

If $R a>M$, acceleration is judged to exist, the final output is

$\theta_{\mathrm{n}}=\theta_{\mathrm{n}-1}+R a$

Where $\theta_{0}$ is output angle, and $\theta_{n-1}$ is the former one. If $R a<M$, the final output is

$\theta_{\mathrm{n}}=Q_{\mathrm{n}}-R a$

then 
$\theta_{\mathrm{n}-1}=\theta_{\mathrm{n}}$

$T_{\mathrm{n}-1}=T_{\mathrm{n}}$

\section{Scheme verification}

Test of the Disturbance Suppresion of Linear Acceleration. Using a shaking table to create linear acceleration in the vertical direction, to observe the final output voltage of the tilt sensor output voltage and MCU DA. Observe the test system of linear acceleration interference suppression effect. Set the parameters to test the linear vibration amplitude of $0.5 \mathrm{~mm}$ when the vibration frequency is $10 \mathrm{~Hz}, 20 \mathrm{~Hz}, 30 \mathrm{~Hz}$; line vibration amplitude of $0.75 \mathrm{~mm}$ when the vibration frequency is $10 \mathrm{~Hz}, 20 \mathrm{~Hz}, 30 \mathrm{~Hz}$; line vibration amplitude of $1.5 \mathrm{~mm}$ vibration frequency $10 \mathrm{~Hz}, 20 \mathrm{~Hz}$, the $30 \mathrm{~Hz}$ acceleration interfere with the output. Elect line vibration $0.75 \mathrm{~mm}-30 \mathrm{~Hz}$, the tilt sensor and microcontroller DA output as shown below.

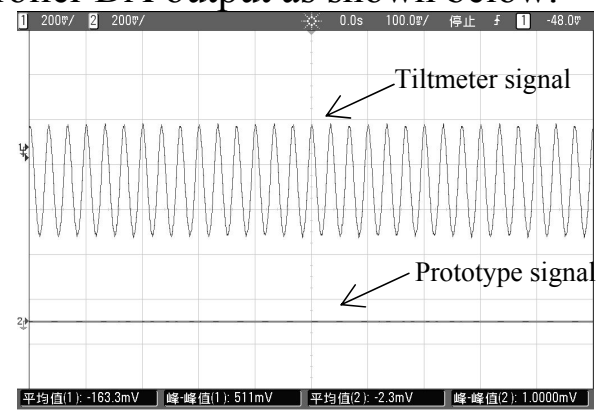

Figure 8. Linear vibration test

From Fig8, the prototype can detect the existence of linear acceleration, and avoid the influence of linear acceleration. Such method cannot guarantee that the linear acceleration in case detection accuracy, because of the integration error increases with the increase in integration time, a long period of continuous integration will inevitably lead to the error increases to unacceptable levels. So the scheme can only be a short period of time to resist the interference of the linear acceleration, to maintain measurement accuracy. Its significance lies in the identification of linear acceleration, and to avoid severe output error angle signal.

Test of suppresion of angular acceleration disturbance.According to the linear relationship shown in Fig.6, the disturbance of angular acceleration can be calculation. The prototype is used to test the suppression of disturbance caused by angular acceleration. Using turntable to make the prototype to vibrate. The parameters of angle vibration is shown in Tab.2. TABLE II. PARAMETER OF TURNTABLE

\begin{tabular}{|l|c|c|}
\hline \multirow{2}{*}{} & \multicolumn{2}{|c|}{ Turntable set } \\
\cline { 2 - 3 } & Angle vibration range & Cycle(s) \\
\hline Case 1 & $-15 \sim 15$ & 5 \\
\hline Case 2 & $-20 \sim 20$ & 5 \\
\hline Case 3 & $-10 \sim 10$ & 4 \\
\hline
\end{tabular}

According to the scale factor of tiltmeter, the tiltmeter voltage should be convert into angle before calculation of RMS(root mean square). The test results are shown in Tab. 3.

$$
R M S=\sqrt{\sum_{i=1}^{N}\left(\theta_{Z i}-\theta_{\mathrm{i}}\right)^{2} / N}
$$

where $N$ is sample number in one period. $\theta_{i}$ is the $(i)$ th data of prototype, $\theta_{\mathrm{zi}}$ is the $(i)$ th data of turntable.

\begin{tabular}{|c|c|c|c|}
\hline TABLE III. ANTI-ANGULAR ACCELERATION DISTURBANCE TEST \\
\hline & Case 1 & Case 2 & Case 3 \\
\hline Before deviation suppression & $0.649^{\circ}$ & $0.675^{\circ}$ & $0.712^{\circ}$ \\
\hline After deviation suppresion & $0.051^{\circ}$ & $0.0469^{\circ}$ & $0.053^{\circ}$ \\
\hline
\end{tabular}


Table 3 shows that the RMS decreased by an degree of magnitude after disturbance suppression. So the angular acceleration compensation algorithm is significantly reduce the disturbance caused by angular acceleration.

\section{Conclusion}

Experiments of the prototype show that the scheme can effectively identify the linear acceleration disturbance, avoid serious error caused by linear acceleration. More importantly, the method to eliminate the disturbance of angular acceleration was verified by angular vibration test. The results of three case show that the scheme can effectively reduce the disturbance of angular acceleration. The scheme cannot resist the disturbance of linear acceleration for a long time, but the elimination of the disturbance caused by angular acceleration is effective when linear acceleration exists.

\section{Acknowledgment}

The work was found by National Natural Science Foundation of China (60971024), Beijing Natural Science Foundation Project(4112020), and Beijing Key Laboratory of Sensor project (5026035209). Thanks a lot.

\section{References}

[1] Zhang Wei-sheng. The Theory And Development Of Tilt Sensor [J]. Sensor World, 2002.8:1821.

[2] Wu Ying, Song Yanhui, Su Weijia. The study about a kind of liquid pendulum angle sensor [J]. Coal Mine Machinery, 2004.6 31-32.

[3] Zhang Fuxue, Mao Xu. Gasflow style level posture sensor and angular velocity gyroscope assembled inertial sensor [J]. Progress in Natural Science, 2007,17(8):971-978.

[4] Yi Ya-xing, LI Zhong-ke, DENG Fang-lin. Research on Inclination Measurement[J]. Computer Automated Measurement \& Control, 2002(06) :360-362.

[5] Piao Lin-hua, Zhang Wei, Zhang Jin-duo, Zhang Fu-xue. Discussion on mechanism of effect of acceleration on gas pendulum tilt sensor[J]. Journal of Transducer Technology, 2004.23(6):4-7 